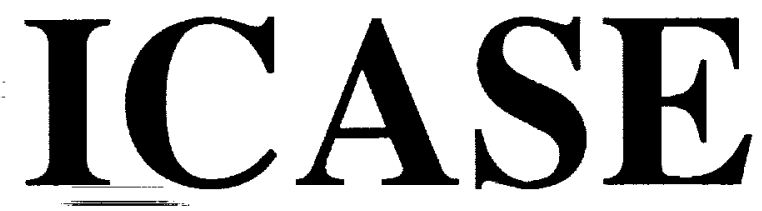

SECONDARY FREQUENCIES IN THE WAKE OF A CIRCULAR CYLINDER WITH VORTEX SHEDDING

\section{Saul S. Abarbanel \\ Wai Sun Don \\ David Gottlieb \\ David H. Rudy \\ James C. Townsend}

Contract Nos. NAS1-18107, NAS1-18605

February 1990

Institute for Computer Applications in Science and Engineering NASA Langley Research Center Hampton, Virginia 23665-5225

Operated by the Universities Space Research Association

\section{N/Sก}

National Aeronautics and

Space Administration

Langley Research Center

Hampton, Virginia 23665-5225

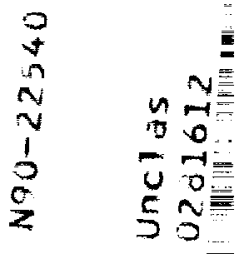

㖶

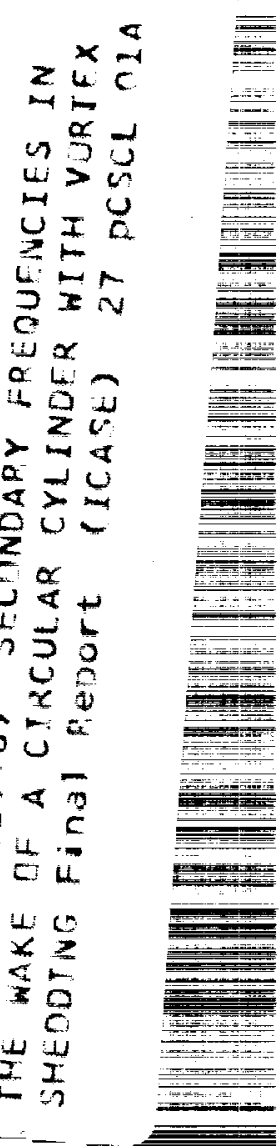

둗 
$\therefore-==\ldots \ldots+\cdots$ 


\title{
Secondary frequencies in the wake of a circular cylinder with vortex shedding*
}

\author{
Saul S. Abarbanel \\ Tel Aviv University, Tel Aviv, Israel \\ Wai Sun Don \\ David Gottlieb \\ Brown University, Providence, RI, USA \\ David H. Rudy \\ James C. Townsend \\ NASA Langley Research Center, Hampton, VA, USA
}

\begin{abstract}
A detailed numerical study of two-dimensional flow past a circular cylinder at moderately low Reynolds numbers has been conducted using three different numerical algorithms for solving the time-dependent compressible Navier-Stokes equations. It was found that if the algorithm and associated boundary conditions were consistent and stable, then the major features of the unsteady wake were well-predicted. However, it was also found that even stable and consistent boundary conditions could introduce additional periodic phenomena reminiscent of the type seen in previous wind-tunnel experiments. However, these additional frequencies were eliminated by formulating the boundary conditions in terms of the characteristic variables. An analysis based on a simplified model provides an explanation for this behavior.
\end{abstract}

\footnotetext{
* Research was supported by the National Aeronautics and Space Administration under NASA Contract Nos. NAS1-18107 and NAS1-18605 while the first three authors were in residence at the Institute for Computer Applications in Science and Engineering (ICASE), NASA Langley Research Center, Hampton, VA 23665.
} 


\section{Introduction}

Historically, the unsteady wake generated by a circular cylinder in low-speed flow has been of great interest to computational fluid dynamicists as well as to theoretical and experimental aerodynamicists. The Reynolds-number range between 40 and 1000 has been of particular interest because it spans the transition from steady flow to unsteady wake flow dominated by the periodic shedding of vortices from the cylinder. The shedding frequency of these vortices increases with Reynolds number over this range, asymptotically approaching a constant value. However, Sreenivasan (1985) measured more than one distinct frequency in the shedding regime at low Reynolds numbers. In addition to the vortex-shedding frequency, he found clearly discernible, lower frequencies in the frequency spectrum for the streamwise velocity measured in the wake. These additional frequencies were not subharmonics of the primary shedding frequency. He concluded that this was a feature of the initial stages of transition to turbulence in agreement with the route to chaos described by Ruelle and Takens (1971). Sirovich (1985) suggested that these additional modes of oscillation could be described theoretically in terms of the classical von Karman vortex street. On the other hand, based on measurements of vibrating and nonvibrating cylinders, van Atta and Gharib (1987) concluded that the additional frequencies found by Sreenivasan were due to the aeroelastic coupling of the vortex wake with cylinder vibration modes. For a nonvibrating cylinder, they found no spectral peaks other than the primary Strouhal shedding frequency. Recently, however, Sreenivasan (1989) determined that the cylinders did not vibrate in his previous experiments.

Conflicting results have also come from the numerical studies of this flow. Karniadakis and Triantafyllou (1989) found no secondary frequency in their computation of the incompressible flow past a circular cylinder using the spectral-element method. Moreover, they were able to excite a secondary mode by introducing an external forcing function into the momentum equations. Townsend et al. (1987) did find a secondary frequency in their finite-difference computation of low-speed compressible flow past a circular cylinder. This low frequency was found in the frequency spectrum of the pressure at various points in the wake. However, the frequency was dependent upon the size of the solution domain, suggesting that it might have been a numerical effect.

The present paper describes the results of further numerical studies which demonstrate that the secondary frequency found in the compressible-flow calculations was produced by 
the far-field boundary conditions. Three different numerical methods were used to solve the time-dependent compressible Navier-Stokes equations. The first of these was the finitedifference technique used by Townsend et al. (1987), the second method was a fully-spectral method (Don 1989), while the third was a mixed spectral-finite-difference method (Don 1989) which was a combination of the other two methods. Computations were made with the finite-difference code with two sets of far-field boundary conditions. These calculations, which were made for flow conditions in both the vortex-shedding regime and the steadywake regime, demonstrate the effect of the boundary conditions on the frequency spectrum of the pressure in the wake. With the proper choice of far-field boundary conditions, no frequencies other than the primary shedding frequency and multiples of the primary frequency were found at a Reynolds number of 80 . In addition, when the "improper" boundary conditions were used to compute the flow at a Reynolds number of 20 , where the flow is known to be steady, then indeed no shedding frequency was predicted although the secondary frequency did appear in the solution. These findings were further substantiated by calculations with the highly-accurate spectral method.

The details of the solution procedures, including boundary conditions, are described in $\S 2$ of the paper. All of the computed results are presented in $\S 3$. In $\S 4$, a mathematical analysis of boundary conditions for a model system is presented which shows how additional frequencies can be produced by the boundary conditions. Finally, a summary of the study and some concluding remarks are given in $\S 5$.

\section{Problem formulation and numerical methods}

\subsection{Governing equations}

For the present compressible-flow calculations, the governing equations are those which describe the conservation of mass, momentum, and energy of an ideal fluid in the absence of external forces. The nondimensional form of these equations in a general curvilinear coordinate system is

$$
\frac{\partial \bar{U}}{\partial t}+\frac{\partial \bar{F}}{\partial \xi}+\frac{\partial \bar{G}}{\partial \eta}=\frac{1}{R e_{r e f}}\left(\frac{\partial \bar{F}_{v}}{\partial \xi}+\frac{\partial \bar{G}_{v}}{\partial \eta}\right)
$$

where 
and

$$
\tau_{y y}=\frac{2}{3} \mu\left[2\left(\frac{\partial \xi}{\partial y} \frac{\partial v}{\partial \xi}+\frac{\partial \eta}{\partial y} \frac{\partial v}{\partial \eta}\right)-\left(\frac{\partial \xi}{\partial x} \frac{\partial u}{\partial \xi}+\frac{\partial \eta}{\partial x} \frac{\partial u}{\partial \eta}\right)\right]
$$

The nondimensional equation of state is

$$
p=(\gamma-1) \rho T
$$

and the viscosity was given by the Sutherland law

$$
\mu=\frac{C_{1} T^{\frac{3}{2}}}{C_{2}+T}
$$

where $C_{1}$ and $C_{2}$ are constants. The ratio of specific heats $\gamma$ was 1.4 , and the Prandtl number $\operatorname{Pr}$ was 0.72 . These equations were nondimensionalized using the following reference quantities: $U_{r e f}=U_{\infty}, \rho_{r e f}=\rho_{\infty}, p_{r e f}=\rho_{\infty} U_{\infty}^{2}$, and $T_{r e f}=U_{\infty}^{2} / c_{v}$ where $U_{\infty}$ and $\rho_{\infty}$ are the free-stream velocity and density, respectively, and $c_{v}$ is the specific heat at constant volume. Therefore, the reference Reynolds number is defined as $R e_{\text {ref }}=U_{\text {ref }} D / \nu_{\text {ref }}$ where $D$ is the diameter of the cylinder and $\nu_{\text {ref }}$ is the kinematic viscosity based on the reference temperature. However, it should be noted that the Reynolds number used in subsequent sections of the paper to characterize the flow conditions for which calculations were made is based upon free-stream quantities, i.e., $R e_{\infty, D}=U_{\infty} D / \nu_{\infty}$.

\subsection{Grid}

A polar grid was used in the calculations. The grid was generated in the physical $(x, y)$ plane and mapped numerically onto the computational $(\xi, \eta)$ plane. The $\xi$ coordinate corresponded to the circumferential direction and the $\eta$ coordinate to the radial direction. The grid was stretched in both directions so that grid points were clustered toward the cylinder in the radial direction and toward the wake region in the circumferential direction. For the finite-difference calculations, the basic grid had 122 points in the circumferential direction and 151 points normal to the body. The outer boundary of the grid was located 25 diameters from the cylinder surface. For the computations with the spectral method, the grid had 64 and 48 points in the angular and radial directions, respectively. Calculations were made with the outer boundary at both 20 and 22.5 diameters away from the cylinder. For computations with the mixed spectral-finite-difference method, the grid had 70 and 


$$
\begin{aligned}
& \bar{U}=\frac{1}{J}\left[\begin{array}{c}
\rho \\
\rho u \\
\rho v \\
E
\end{array}\right] \\
& \bar{F}=\frac{F}{J} \frac{\partial \xi}{\partial x}+\frac{G}{J} \frac{\partial \xi}{\partial y}, \quad \bar{G}=\frac{F}{J} \frac{\partial \eta}{\partial x}+\frac{G}{J} \frac{\partial \eta}{\partial y}, \\
& F=\left[\begin{array}{c}
\rho u \\
\rho u^{2}+p \\
\rho u v \\
(E+p) u
\end{array}\right], \quad G=\left[\begin{array}{c}
\rho v \\
\rho u v \\
\rho v^{2}+p \\
(E+p) v
\end{array}\right] \\
& \bar{F}_{v}=\frac{F_{v}}{J} \frac{\partial \xi}{\partial x}+\frac{G_{v}}{J} \frac{\partial \xi}{\partial y}, \quad \bar{G}_{v}=\frac{F_{v}}{J} \frac{\partial \eta}{\partial x}+\frac{G_{v}}{J} \frac{\partial \eta}{\partial y}, \\
& F_{v}=\left[\begin{array}{c}
0 \\
\tau_{x x} \\
\tau_{x y} \\
\frac{\gamma}{P r} \kappa \frac{\partial T}{\partial x}+u \tau_{x x}+v \tau_{x y}
\end{array}\right], \quad G_{v}=\left[\begin{array}{c}
0 \\
\tau_{x y} \\
\tau_{y y} \\
\frac{\gamma}{P r} \kappa \frac{\partial T}{\partial \nu}+u \tau_{x y}+v \tau_{v y}
\end{array}\right] \text {, }
\end{aligned}
$$

$J$ is the Jacobian of the transformation from Cartesian coordinates $(\mathrm{x}, \mathrm{y})$ to the general curvilinear coordinates $(\xi, \eta)$,

$$
J=\frac{\partial \xi}{\partial x} \frac{\partial \eta}{\partial y}-\frac{\partial \xi}{\partial y} \frac{\partial \eta}{\partial x}
$$

and the specific internal energy, $E$, is defined by

$$
E=\rho\left[T+\frac{1}{2}\left(u^{2}+v^{2}\right)\right] .
$$

The elements of the stress tensor are

$$
\begin{gathered}
\tau_{x x}=\frac{2}{3} \mu\left[2\left(\frac{\partial \xi}{\partial x} \frac{\partial u}{\partial \xi}+\frac{\partial \eta}{\partial x} \frac{\partial u}{\partial \eta}\right)-\left(\frac{\partial \xi}{\partial y} \frac{\partial v}{\partial \xi}+\frac{\partial \eta}{\partial y} \frac{\partial v}{\partial \eta}\right)\right] \\
\tau_{x y}=\mu\left[\left(\frac{\partial \xi}{\partial y} \frac{\partial u}{\partial \xi}+\frac{\partial \eta}{\partial y} \frac{\partial u}{\partial \eta}\right)+\left(\frac{\partial \xi}{\partial x} \frac{\partial v}{\partial \xi}+\frac{\partial \eta}{\partial x} \frac{\partial v}{\partial \eta}\right)\right]
\end{gathered}
$$


100 points in the circumferential and radial directions, respectively. For this case, the farfield boundary was located 22.5 diameters from the cylinder surface. In the experiments of Sreenivasan (1985), the wind-tunnel walls were located 30 diameters above and below the cylinder ${ }^{1}$.

\subsection{Numerical methods}

The finite-difference method used in the present study was the original unsplit technique of MacCormack (1969), which has second-order accuracy in both space and time. The method is an explicit, conditionally-stable, predictor-corrector scheme. Forward differences were used to approximate the derivatives of the fluxes in equation (2.1) in the predictor step, and backward differences were used in the corrector step. The first derivatives appearing in the viscous flux terms, $F_{v}$ and $G_{v}$, were approximated with backward differences if the flux derivative was being approximated with a forward difference, and vice-versa.

The spectral algorithm used both the Fourier and Chebyshev collocation methods. Because of the polar grid, the flow could be treated as being periodic at the boundaries of the solution domain in the circumferential direction. Thus, the Fourier collocation (pseudospectral) method was the natural choice for the circumferential direction. Since the flow in the radial direction was not periodic, the Chebyshev collocation method was used in that direction. Both of these methods were employed in the form of the matrix vector multiplication method instead of the more commonly used Fast Fourier Transform method. To improve the stability of the algorithm, a fourth-order exponential filter (Don 1989) was applied to the differentiation and solution matrices. The solution was advanced in time using a second-order Runge-Kutta method.

A third approach which combined these two methods was also used. In this case, the Fourier method was used in the circumferential direction and the finite-difference method in the radial direction.

\subsection{Boundary conditions}

A sketch of the solution domain in the physical plane is shown in figure 1. Periodic boundary conditions for the dependent variables were applied along the cut line in the

\footnotetext{
${ }^{1}$ Private communication.
} 
wake for both the finite-difference and spectral methods. Standard solid-wall boundary conditions for viscous flow were applied at the cylinder surface. A no-slip condition was applied so that the velocity components, $u$ and $v$, were specified to be zero at the surface. The wall temperature was held fixed at the value of the free-stream temperature. In the finite-difference code, the density at the wall was obtained from an extrapolation of interior-point values of pressure in the direction normal to the wall. In the spectral code, the density at the wall was computed as a part of the solution.

The outer boundary is an arbitrarily-chosen boundary, introduced only to restrict the computational domain to a finite size. Since this is a boundary across which fluid passes either into or out of the computational region and since disturbances can propagate upstream as well as downstream in a subsonic flow, careful consideration must be given to the specification of boundary conditions on this outer boundary. If this outer boundary is located sufficiently far from the cylinder surface, viscous effects are negligible in the flow crossing the boundaries except in the narrow region where the cylinder wake is located. As a result, the proper choice of boundary conditions can be found from an analysis of the inviscid form of the governing equations.

For a subsonic inflow boundary, this analysis shows that there are three characteristics coming into the solution domain and one outgoing characteristic. Thus, there must be three quantities specified at an inflow boundary. Since there are four governing equations, the numerical method requires a fourth boundary condition in addition to the three required for proper specification of the boundary conditions in a mathematical sense. In the finite-difference code, calculations were made with two different sets of inflow boundary conditions. The first set, which was used in the previous solutions reported by Townsend et al. (1987), was formulated in terms of the primitive variables $u, v, \rho$, and $T$. At the inflow boundary, free-stream values of the two velocity components and the temperature were specified, i.e.,

$$
\begin{aligned}
& u_{\text {inflow }}=u_{\infty} \\
& v_{\text {inflow }}=v_{\infty} \\
& T_{\text {inflow }}=T_{\infty}
\end{aligned}
$$

The density was obtained from a zeroth-order extrapolation of pressure from the interior of the solution domain and the use of equation (2.7). In preliminary calculations in which 
only the primary shedding frequency was of interest, this boundary condition gave results which were not significantly different from those obtained using an extrapolation of the outgoing characteristic to obtain pressure.

At the outflow boundary, only one analytic boundary condition is needed since there is only one incoming characteristic. A typical choice is the specification of the static pressure. However, as demonstrated numerically by Rudy and Strikwerda (1980), this can cause waves to be reflected from the outflow boundary back into the solution domain, adversely affecting the solution in the interior of the domain. A nonreflecting boundary condition based on the work of Engquist and Majda (1977) was used in the present study. Thus, the pressure at the outflow boundary was found from a finite-difference approximation to the equation

$$
\frac{\partial p}{\partial t}-\rho c\left(\frac{\partial u}{\partial t}-u_{\infty} \frac{\partial v}{\partial y}\right)=0
$$

where $c$ is the nondimensional speed of sound given by

$$
c=\sqrt{\gamma(\gamma-1) T}
$$

This boundary condition was applied along the outflow boundary where the viscous wake crossed the boundary as well as the region immediately above and below the wake. Along the remainder of the outflow boundary, where the flow was essentially inviscid, the pressure was specified to be the free-stream pressure. The variables $u, v$, and $T$ along the entire outflow boundary were obtained from zeroth-order extrapolation, and the density was then obtained from equation (2.7) using the boundary value of $p$ and the extrapolated value of $T$.

The second set of boundary conditions, which was also used in the spectral calculations, was based entirely on the characteristic variables. An analysis of the inviscid form of equation (2.1), linearized around the free-stream conditions, shows (Gottlieb, Lustman, and Streett 1984) that the characteristic variables, with corresponding eigenvalues $a_{1}=$ $a_{2}=\vec{U} \cdot \vec{N}, a_{3}=\vec{U} \cdot \vec{N}-c$, and $a_{4}=\vec{U} \cdot \vec{N}+c$, are

$$
\begin{aligned}
& R_{1}=\rho \frac{c_{\infty}^{2}}{\gamma-1}-\left(\frac{1}{2} \rho \vec{U}_{\infty} \cdot \vec{U}_{\infty}-\vec{M} \cdot \vec{U}_{\infty}+E\right) \\
& R_{2}=\left(m_{u}-\rho u_{\infty}\right) \tilde{\eta}_{y}-\left(m_{v}-\rho v_{\infty}\right) \tilde{\eta}_{x} \\
& R_{3}=-\left(\vec{M}-\rho \vec{U}_{\infty}\right) \cdot \vec{N}+\left(\frac{\gamma-1}{c_{\infty}}\right)\left(\frac{1}{2} \rho \vec{U}_{\infty} \cdot \vec{U}_{\infty}-\vec{M} \cdot \vec{U}_{\infty}+E\right) \\
& R_{4}=\left(\vec{M}-\rho \vec{U}_{\infty}\right) \cdot \vec{N}+\left(\frac{\gamma-1}{c_{\infty}}\right)\left(\frac{1}{2} \rho \vec{U}_{\infty} \cdot \vec{U}_{\infty}-\vec{M} \cdot \vec{U}_{\infty}+E\right)
\end{aligned}
$$


where $\vec{U}_{\infty}=\left(u_{\infty}, v_{\infty}\right)$ is the free-stream velocity, $\vec{M}=\left(m_{u}, m_{v}\right)=(\rho u, \rho v)$ is the local momentum, $c_{\infty}$ is the free-stream speed of sound, and $\vec{N}=\left(\tilde{\eta}_{x}, \tilde{\eta}_{y}\right)=\left(\frac{\partial \eta}{\partial x}, \frac{\partial \eta}{\partial y}\right) / \sqrt{\left(\frac{\partial \eta}{\partial x}\right)^{2}+\left(\frac{\partial \eta}{\partial y}\right)^{2}}$ is the unit outward normal vector. At the subsonic inflow boundary, the characteristic variables corresponding to the three incoming characteristics were specified using free-stream values. These boundary values then became .

$$
F_{i}=R_{i}\left(\rho_{\infty}, \vec{M}_{\infty}, E_{\infty}\right), \quad i=1,2,3
$$

Furthermore, the required numerical boundary condition is

$$
F_{4}=R_{4}\left(\rho_{n u m}, \vec{M}_{n u m}, E_{n u m}\right)
$$

where values in $F_{4}$ are obtained by extrapolation from the interior of the domain in the finite-difference code and are obtained as a part of the solution in the spectral method. Similarly, at the outflow boundary, the characteristic variable corresponding to the incoming characteristic was specified, i.e.,

$$
F_{3}=R_{3}\left(\rho_{\infty}, \vec{M}_{\infty}, E_{\infty}\right)
$$

and the values for the other three characteristic variables were found numerically as at the inflow boundary, so that

$$
F_{i}=R_{i}\left(\rho_{n u m}, \vec{M}_{n u m}, E_{n u m}\right), \quad i=1,2,4
$$

At each boundary, the conserved variables were then computed from the appropriate system of equations, giving

$$
\begin{aligned}
\rho & =\frac{\gamma-1}{c_{\infty}^{2}}\left(F_{1}+\phi_{1}\right) \\
m_{u} & =\left(\phi_{2} \tilde{\eta_{x}}+\phi_{3} \tilde{\eta_{y}}\right)+\rho u_{\infty} \\
m_{v} & =\left(\phi_{2} \tilde{\eta_{v}}-\phi_{3} \tilde{\eta_{x}}\right)+\rho v_{\infty} \\
E & =\phi_{1}-\frac{1}{2} \rho \vec{U}_{\infty} \cdot \vec{U}_{\infty}+m_{u} u_{\infty}+m_{v} v_{\infty}
\end{aligned}
$$

on the outer boundary, where

$$
\phi_{1}=\frac{c_{\infty}}{\gamma-1} \frac{\left(F_{3}+F_{4}\right)}{2}, \quad \phi_{2}=\frac{1}{2}\left(F_{4}-F_{3}\right), \quad \phi_{3}=F_{2} .
$$




\section{Results and discussion}

Computations were made with all three methods for Mach 0.4 flow at a Reynolds number of 80 . The initial flow field was specified to be free-stream flow everywhere except at the cylinder surface. Figure 2(a) shows pressure contours (isobars) from the finitedifference solution using the primitive-variable far-field boundary conditions at a point in time after the periodic vortex shedding had been fully established. The pressure field for finite-difference solution using the characteristic far-field boundary conditions is shown in figure 2(b). The corresponding solution for the fully-spectral code is given in figure 3.

As the computations were made, the values of the pressure were saved at ten selected grid points in the flow field. At least four of these grid points were located in the wake region and the others were upstream of the cylinder. For the finite-difference code, the computed pressure data were saved every twenty time steps, and for the fully-spectral and mixed spectral-finite-difference codes, because of the larger time step used in these methods, the data were saved after every time step.

The set of pressure data at each point was analyzed for its frequency spectrum using a discrete Fast Fourier Transform (FFT) technique. First, the time-averaged pressure was subtracted from the pressure data to form a time sequence $p_{j}$ of $N$ points, where $N=2^{m}$ for some positive integer $m$. Second, the Fourier coefficients $F_{k}$ of the transformation

$$
F_{k}=A \sum_{j=0}^{N-1} p_{j} e^{-i\left(\frac{2 \pi j k}{N}\right)} \text { for } k=0,1, \ldots, N-1
$$

were obtained using a pre-coded FFT subroutine. $A=1$ in the subroutine used to analyze the finite-difference results and $A=1 / N$ in the subroutine used to analyze the results from the fully-spectral and mixed-method codes. The power spectral density $d_{k}$ for wave number $k$ was found then simply as

$$
d_{k}=B\left|F_{k}\right|^{2} \text { where } k=0,1, \ldots, N-1 .
$$

$B=2 / N$ for the finite-difference results and $B=1$ for the others. The Strouhal number (nondimensional frequency) corresponding to the vortex-shedding frequency was

$$
S t_{1}=k_{1} /(N \Delta t)
$$

where $\Delta t$ was the time step and $k_{1}$ was the value of $k$ such that

$$
d_{k_{1}}=\max _{k} d_{k} .
$$


Similarly, $S t_{2}$ is the Strouhal number corresponding to the secondary frequency.

Note that, because the power density was found only for discrete frequencies, there was an inherent discretization error in determining the dominant frequencies in the flow. The actual peak frequency would be within half of the discretization, or $\pm 1 /(2 N \Delta t)$, of that given by the FFT. In some of the present cases, the resulting error in the Strouhal number could be as large as 0.003 . In an effort to get a somewhat better estimate, for each dominant wave number a peak in the frequency spectrum was estimated using a threepoint parabolic interpolation. These values were used in table 1 , which summarizes the results found in the present study.

\begin{tabular}{|c|c|c|c|c|c|c|c|}
\hline Method & $R e_{\infty, D}$ & $M_{\infty}$ & Domain & Inflow BC & Outflow BC & $S t_{1}$ & $S t_{2}$ \\
\hline \hline FD & 80 & 0.4 & $51 D$ & Primitive & Primitive & .1515 & .0165 \\
FD & 80 & 0.2 & $51 \mathrm{D}$ & Primitive & Primitive & .1504 & .0347 \\
FD & 80 & 0.1 & $51 \mathrm{D}$ & Primitive & Primitive & .1564 & .0721 \\
FD & 80 & 0.4 & 51D & Characteristic & Characteristic & .1518 & - \\
S & 80 & 0.4 & $46 \mathrm{D}$ & Characteristic & Characteristic & .1574 & - \\
S & 80 & 0.4 & $41 \mathrm{D}$ & Characteristic & Characteristic & .1565 & - \\
M & 80 & 0.4 & $46 \mathrm{D}$ & Primitive & Primitive & .1589 & .0225 \\
M & 80 & 0.4 & 46D & Characteristic & Characteristic & .1558 & - \\
\hline
\end{tabular}

Table 1: Shedding frequencies computed with finite-difference code (FD), mixed finite-difference-spectral code (M), and fully-spectral code (S).

These frequencies were based on the computed pressure at a point in the wake $10 \mathrm{di}$ ameters downstream of the cylinder and one diameter above the wake centerline. This location corresponds to the principle one used in the experiments of Sreenivasan (1985). It should be noted that the frequencies for the finite-difference solution with primitive variables at Mach 0.4 differ slightly from those given by Townsend et al. (1987), in which the results were incorrect due to a misinterpretation of the output from the FFT subroutine.

Experimental measurements of the primary shedding frequency in the wake of a circular cylinder at low Reynolds numbers were reported by Roshko (1953). An approximation to 
the data was given by the equation

$$
S t_{1}=0.212\left(1-\frac{21.2}{R e_{\infty, D}}\right)
$$

For a Reynolds number of 80 , this equation yields $S t_{1}=0.1558$. As shown in table 1 , the computed values of $S t_{1}$ are very close to the experimental value in all cases. The largest deviation is $3.47 \%$ and most of the values are within $2 \%$ of the experimental frequency. Therefore, the primary vortex-shedding frequency in the wake is well-predicted with either set of boundary conditions.

Plots of the time history of the pressure and the resulting frequency spectrum at the selected point in the wake flow from the finite-difference calculations are shown in figure 4 for both sets of boundary conditions. The corresponding plots for the fully-spectral calculations with characteristic boundary conditions are shown in figure 5. As shown in figure 4, the solution with primitive-variable boundary conditions produces a low frequency which is not seen in the spectrum from the calculations with the characteristic boundary conditions. This frequency is not a subharmonic of the primary shedding frequency and was not found in any of the solutions using any of the three codes when characteristic boundary conditions were employed. Furthermore, the amplitude of the secondary frequency remained relatively constant at all locations for which the spectrum was computed, including a location 10 diameters upstream of the cylinder. At this point, the amplitude of the primary shedding frequency was significantly lower than in the wake so that the secondary frequency was the dominant frequency in the signal. These factors suggest that the secondary frequency is a spurious numerical artifact.

Calculations were also made with the finite-difference code using the primitive-variable boundary conditions to determine the effect of Mach number on the computed frequencies. Computations were made at Mach numbers of 0.2 and 0.1 for a Reynolds number of 80 . As shown in table 1 , there was only a small effect on $S t_{1}$ as Mach number was lowered. However, as Mach number was decreased by a factor of $2, S t_{2}$ increased by a factor of approximately 2 .

To further establish that the secondary frequency is a numerical artifact, calculations were made for Mach 0.4 flow at a Reynolds number of 20 . Under these conditions, the flow in the wake should be steady, consisting of two symmetric counterrotating vortices just behind the cylinder. Calculations with the finite-difference code produced the expected 
steady wake with both sets of boundary conditions. However, when the primitive-variable boundary conditions were used, a secondary frequency appeared in the frequency spectrum of the pressure in the wake with a value of $S t_{2}=0.0158$. This spurious secondary frequency did not appear when characteristic boundary conditions were used.

In summary, the calculations presented in this section demonstrate that a secondary frequency can be introduced into the solution by the far-field boundary conditions. By coincidence, the value of this frequency was very close to that found experimentally by Sreenivasan (1985). This frequency was not found when the boundary conditions are properly formulated in terms of characteristic variables.

\section{Analysis}

As shown in $\S 3$, the secondary frequency disappeared when boundary conditions based on the characteristic variables were used at the outer boundary, demonstrating that the secondary frequency computed with the primitive-variable boundary conditions was, in fact, of numerical origin. In this section, a theoretical explanation is given for these results using a modal analysis of the effect of inflow boundary conditions on the stability of small perturbations to the flow. It is shown that the use of boundary conditions based on noncharacteristic variables causes a temporally-periodic perturbation. Furthermore, it is demonstrated that the use of characteristic variables eliminates this periodic disturbance.

The theoretical study is based upon a one-dimensional treatment of the Euler equations. The use of the inviscid form of the governing equations is justified at distances far from the cylinder since the Reynolds numbers under consideration place the flow well outside the limits of the Stokes and Oseen regions. The assumption of one-dimensionality is more problematical. However, it is a reasonably good representation for the calculations in which a finite-difference method was used in the radial direction and the Fourier collocation method was used in the circumferential direction. In fact, it will be shown that the theoretical predictions of the secondary frequency are in good agreement (within 15\%) with the computed values for this case. The agreement with the computed results from the fully finite-difference code is not as good, although the trend with Mach number is well-predicted.

The analysis considers a region of flow along the $\mathrm{x}$ axis sufficiently far upstream from the cylinder. The one-dimensional form of equation (2.1), under the assumption of inviscid 
flow then becomes

$$
\frac{\partial U}{\partial t}+\frac{\partial F}{\partial x}=0
$$

where

$$
\begin{gathered}
U=\left[\begin{array}{c}
\rho \\
m \\
E
\end{array}\right], \\
F=\left[\begin{array}{c}
m \\
\frac{m^{2}}{p}+p \\
u(E+p)
\end{array}\right],
\end{gathered}
$$

and $m=\rho u$. In terms of the dependent variables in $U$, the equation of state, equation (2.7), is

$$
p=(\gamma-1)\left[E-\frac{1}{2}\left(\frac{m^{2}}{\rho}\right)\right]
$$

Equation (4.1) can be rewritten in nonconservation form as

$$
\frac{\partial U}{\partial t}+A(U) \frac{\partial U}{\partial x}=0
$$

where $A(U)=\frac{\partial F}{\partial U}$ is the Jacobian of the flux with respect to the solution vector $U$. Linearizing about the steady free-stream conditions, this equation becomes

$$
\frac{\partial}{\partial t}(\delta U)+A\left(U_{\infty}\right) \frac{\partial}{\partial x}(\delta U)=0
$$

where $\delta U=U-U_{\infty}$ is the perturbation vector. The matrix $A\left(U_{\infty}\right)=\left[\frac{\partial F(U)}{\partial U}\right]_{U=U_{\infty}}$ has three eigenvalues $a_{1}=u_{\infty}-c_{\infty}, a_{2}=u_{\infty}+c_{\infty}$, and $a_{3}=u_{\infty}$. The corresponding eigenvectors in terms of the conserved-variable perturbations are

$$
\begin{aligned}
& R_{1}=-\left(\delta m-u_{\infty} \delta \rho\right)+\frac{\gamma-1}{c_{\infty}}\left[\frac{1}{2} u_{\infty}^{2} \delta \rho-u_{\infty} \delta m+\delta E\right] \\
& R_{2}=\left(\delta m-u_{\infty} \delta \rho\right)+\frac{\gamma-1}{c_{\infty}}\left[\frac{1}{2} u_{\infty}^{2} \delta \rho-u_{\infty} \delta m+\delta E\right]
\end{aligned}
$$

and

$$
R_{3}=c_{\infty} \delta \rho-\frac{\gamma-1}{c_{\infty}}\left[\frac{1}{2} u_{\infty}^{2} \delta \rho-u_{\infty} \delta m+\delta E\right]
$$


Furthermore, using a linearized version of the equation of state, i.e.,

$$
\delta p=(\gamma-1)\left[\frac{1}{2} u_{\infty}^{2} \delta \rho-u_{\infty} \delta m+\delta E\right],
$$

equations (4.5)-(4.7) may also be written in terms of the primitive-variable perturbations $(\delta p, \delta u, \delta \rho)$, giving,

$$
\begin{aligned}
& R_{1}=-\rho_{\infty} \delta u+\frac{1}{c_{\infty}} \delta p \\
& R_{2}=\rho_{\infty} \delta u+\frac{1}{c_{\infty}} \delta p
\end{aligned}
$$

and

$$
R_{3}=c_{\infty} \delta \rho-\frac{1}{c_{\infty}} \delta p .
$$

Any perturbation imposed on the free-stream solution will evolve as a combination of these eigenvectors. This evolution can be studied locally near the outer computational boundary by employing the modal analysis developed by Gustafsson, Kreiss, and Sundström (1972) and Osher (1969) to study the stability of numerical approximations to initial-boundaryvalue problems.

Consider an inflow boundary point with subsonic flow crossing the boundary into the solution domain. At this point, $a_{2}=u_{\infty}+c_{\infty}>0$ and $a_{3}=u_{\infty}>0$. Therefore, the characteristic variables, $R_{2}$ and $R_{3}$, corresponding to these two incoming characteristics must be specified. The third one, $R_{1}$, corresponds to $a_{1}=u_{\infty}-c_{\infty}<0$ and thus exits the domain. To model the situation near the inflow boundary, consider the governing equations in terms of the characteristic variables, viz.,

$$
\begin{aligned}
\frac{\partial R_{1}}{\partial t}+\left(u_{\infty}-c_{\infty}\right) \frac{\partial R_{1}}{\partial x} & =0 \\
\frac{\partial R_{2}}{\partial t}+\left(u_{\infty}+c_{\infty}\right) \frac{\partial R_{2}}{\partial x} & =0 \\
\frac{\partial R_{3}}{\partial t}+u_{\infty} \frac{\partial R_{3}}{\partial x} & =0
\end{aligned}
$$

or

$$
\frac{\partial R_{s}}{\partial t}+a_{s} \frac{\partial R_{s}}{\partial x}=0, \quad s=1,2,3 .
$$

In the one-dimensional case, the finite-difference algorithm described in $\S 3$ is equivalent to a Lax-Wendroff scheme and can be written as

$$
w_{j}^{s, n+1}=w_{j}^{s, n}-\frac{a_{s} \Delta t}{2 \Delta x}\left(w_{j+1}^{s, n}-w_{j-1}^{s, n}\right)+\frac{a_{s}^{2}(\Delta t)^{2}}{2(\Delta x)^{2}}\left(w_{j+1}^{s, n}-2 w_{j}^{s, n}+w_{j-1}^{s, n}\right), \quad j>0,
$$


where $w_{j}^{s, n}=w^{s}(j \Delta x, n \Delta t)$ is the finite-difference approximation to $R_{s}(x, t)$. Equation (4.16) is solved with inflow-type boundary conditions imposed at $x_{b o u n d a r y}=(j \Delta x)_{j=0}$ which are of the form

$$
\begin{aligned}
& \left(R_{2}-\alpha R_{1}\right)_{j=0}=0, \\
& \left(R_{3}-\beta R_{1}\right)_{j=0}=0,
\end{aligned}
$$

and

$$
\left(R_{1}+\sigma R_{2}+\epsilon R_{3}\right)_{j=0}=\left(R_{1}+\sigma R_{2}+\epsilon R_{3}\right)_{j=1} .
$$

Thus, equation (4.19) is a zeroth-order extrapolation on $R_{1}$.

The modal solution ansatz for this case is

$$
R_{s}=A_{s} \kappa_{s}^{j} z^{n} \quad s=1,2,3
$$

Substituting this solution into equation (4.16) yields the three modal characteristic equations given by

$$
\left(\lambda_{s}^{2}-\lambda_{s}\right) \kappa_{s}^{2}+2\left(1-z-\lambda_{s}^{2}\right) \kappa_{s}+\left(\lambda_{s}^{2}+\lambda_{s}\right)=0, \quad s=1,2,3
$$

where $\lambda_{s}=\frac{a_{s} \Delta t}{\Delta x}$. Substituting equation (4.20) into equations (4.17) to (4.19) gives the modal representation of the boundary conditions,

$$
\begin{aligned}
& A_{2}=\alpha A_{1}, \quad\left(A_{1} \neq 0\right) \\
& A_{3}=\beta A_{1}
\end{aligned}
$$

and

$$
A_{1}\left(1-\kappa_{1}\right)+\sigma A_{2}\left(1-\kappa_{2}\right)+\epsilon A_{3}\left(1-\kappa_{3}\right)=0 .
$$

Since $A_{1} \neq 0$, equation (4.24) may be rewritten as

$$
\left(1-\kappa_{1}\right)+\alpha \sigma\left(1-\kappa_{2}\right)+\beta \epsilon\left(1-\kappa_{3}\right)=0 .
$$

It is necessary to find $\mathrm{z}$ such that $|z|=1$ and $\kappa_{s}(s=1,2,3)$ such that $\left|\kappa_{s}\right|<1$ which solve equations (4.21) and (4.25) simultaneously. It should be noted that $|z|=1, z \neq 1$, corresponds to a solution that is purely periodic in time. If the search for such solutions fails 
(which it does, as it will be shown, when $\alpha=\beta=0$, i.e., for characteristic specification), then the boundary treatment does not introduce any spurious frequency. If, under a given set of boundary conditions, there is a solution to equations (4.21) to (4.25), then the phase of $z$ gives the temporal frequency of the numerically-introduced perturbation. Of course, it is hoped that at least one of the $\kappa$ 's will be nearly 1 in magnitude. Otherwise, the temporally-periodic perturbation will decay spatially as $|\kappa|^{j}$. In fact, in all of the calculations with the model, it is always the case that $.995<\left|\kappa_{2}\right|<1$.

In the actual numerical computations presented in $\S 3$, the noncharacteristic boundary conditions (translated to the present one-dimensional model) consisted of specifying the velocity and temperature $\left((\delta u)_{j=0}=0,(\delta T)_{j=0}=0\right)$ and performing the extrapolation

$$
(\rho)_{j=0}=(\rho)_{j=1} \frac{T_{j=1}}{T_{\infty}}
$$

This is equivalent to

$$
\begin{aligned}
(\delta \rho)_{j=0} & =\left(\frac{T_{1}}{T_{\infty}}\right)(\delta \rho)_{j=1}+\frac{T_{1}-T_{\infty}}{T_{\infty}} \rho_{\infty} \\
& =\left(\frac{T_{1}}{T_{\infty}}\right)(\delta \rho)_{j=1}+\frac{\rho_{\infty}}{T_{\infty}}(\delta T)_{j=1} .
\end{aligned}
$$

This is not a "pure" extrapolation on the density. Using $(\delta u)_{j=0}=(\delta T)_{j=0}=0$ in equations (4.17) and (4.18) leads directly to $\alpha=1$ and $\beta=\gamma-1$. If equation (4.27) were a pure extrapolation on $\rho$, then from the relation

$$
2 c_{\infty} \delta \rho=R_{1}+R_{2}+2 R_{3}
$$

(see equations (4.9) to (4.11)) it would follow that $\sigma=1$ and $\epsilon=2$. However, it should be noted that while $\delta u$ does not appear in equation (4.27), and hence $\sigma=1$ in (4.19), $\epsilon$ must have a value which depends on the solution inside the domain. Therefore, it is necessary to seek solutions to the nonlinear system (4.21) and (4.25) for a given $\lambda_{\text {s }}$ such that $|z|=1, z \neq 1,\left|\kappa_{z}\right|<1$, and $\alpha \sigma=1$. The solution that satisfies $z=e^{i \theta}(\theta \neq 0)$, $\left|\kappa_{s}\right|<1(s=1,2,3)$ with $\alpha \sigma=1$ then yields the value of $\epsilon \beta=(\gamma-1) \epsilon$. The numerical solution of equations (4.21) and (4.25) can be sensitive near $\left|\kappa_{s}\right|=1$ so double precision was used in the numerical solutions of these equations. For a wide range of $M_{\infty}$ (and hence $\lambda$ ), it was found that $\epsilon \beta=-1.24 \pm 0.2$, giving confidence in the model ${ }^{2}$. The $\lambda$ used in

\footnotetext{
${ }^{2}$ In fact, it can be easily shown that the model boundary conditions (4.17)-(4.19), with $\alpha=\sigma=1$, $\beta=\gamma-1$, and $\epsilon$ found from the solution to (4.21) to (4.25), correspond to a primitive-variable boundary condition of the form $(\rho)_{j=0}=(\rho)_{j=1} \frac{T_{j=1}}{T_{\rho}}+a\left(\Delta r_{\max }\right)^{2+m}$ where $a=O(1)$ and $m \geq 0$. Thus, the analysis is performed on a boundary condition which, within the second-order accuracy of the finite-difference code, cannot be numerically distinguished from the one actually imposed i.e., equation (4.26).
} 
the theoretical prediction, $\lambda=(u+c) \frac{\Delta t}{\Delta x}$, was based on the actual time step used in the Navier-Stokes codes and $\Delta x$ was taken as the radial cell size $\left(\Delta r_{\max }\right)$ nearest to the inflow boundary.

The present theoretical model most closely corresponds to the Navier-Stokes code with the finite-difference method in the radial direction and the Fourier collocation method in the circumferential direction. A comparison of the theoretical Strouhal number from the one-dimensional model with the one obtained from this Navier-Stokes code is given in Table 2. In view of the fact that the analysis is based on a one-dimensional model, the agreement between the values of $S t_{2}$ predicted by the model and the computed values is reasonably good. Furthermore, the model predicts the trend of decreasing $S t_{2}$ with increasing domain size.

\begin{tabular}{|c|c|c|c|c|c|}
\hline $\mathrm{N}$ & $\Delta r_{\max }$ & $\Delta t_{\text {code }}$ & $\lambda_{\text {model }}$ & $\left(S t_{2}\right)_{\text {code }}$ & $\left(S t_{2}\right)_{\text {model }}$ \\
\hline \hline 100 & .41 & .0053 & .0452 & .0244 & .0296 \\
100 & .46 & .0053 & .0403 & .0225 & .0264 \\
\hline
\end{tabular}

Table 2: Comparison of secondary frequencies predicted by the analysis and computed using the mixed spectral-finite-difference code. $M_{\infty}=0.4$.

Finally, it is seen that if the characteristic boundary conditions, (i.e., $\alpha=\beta=0$ in equations (4.17) to (4.19)) are used, then the only solution to (4.21) through (4.25) is $A_{2}=A_{3}=0, \kappa_{1}=1$, and $z=1$. It can be shown by perturbation analysis that it is not a generalized eigenvalue. Thus, any perturbation from the boundary, $R_{1}=A_{1} \kappa_{1}^{j} z^{n}=A_{1}$ will remain constant and small.

\section{Concluding remarks}

A numerical study has been conducted using three different codes to compute the unsteady flow about a circular cylinder in low-speed flow at low Reynolds numbers. One of these codes used a finite-difference method to solve the two-dimensional time-dependent compressible Navier-Stokes equations, the second used spectral techniques, and the third used a combination of these two methods. With stable and consistent boundary conditions, all of these methods were able to predict accurately the major features of the flow such as the vortex-shedding frequency. However, it was found that certain far-field boundary 
conditions which used extrapolation of the primitive variables introduced an additional temporal frequency into the solution. By coincidence, the value of this frequency was very close to that found experimentally by Sreenivasan (1985). The use of characteristic variables in the far-field boundary conditions eliminated this frequency from the solution. An explanation of this behavior was provided using an analysis based on a simplified model for the boundary conditions. These results illustrate the fact that great care must be taken in interpreting the results from numerical simulations, and that while the secondary frequencies are spurious in the infinite-domain case, the possibility exists that they may be found in a confined space such as a wind tunnel. In fact, Sreenivasan ${ }^{3}$ found that the secondary frequency disappeared when the tests were performed in a wind tunnel where the distance from the cylinder to the upper and lower tunnel walls was 250 cylinder diameters. This distance is about seven times that used in the original tests. Insight into the presence of the multiple frequencies in the original experimental results would require three-dimensional computations which included the wind-tunnel walls.

\section{REFERENCES}

DoN, W. S. 1989 Theory and application of spectral methods for the unsteady compressible wake flow past a two-dimensional circular cylinder. PhD Thesis. Brown University.

ENQUIST,B. \& MAJDA, A. 1977 Absorbing boundary conditions for the numerical simulation of waves. Math. Comp. 31, 629-651.

GotTlieb,D., Lustman, L., \& StReETt,C.S. 1984 Spectral methods for two-dimensional shocks. In Spectral Methods for Partial Differential Equations (ed. R. G. Voigt, D. Gottlieb, \& M. Y. Hussaini), 79-95. SLAM-CBMS.

Gustafsson, B., KREISS, H.-O., \& SUNDSTRöM,A. 1972 Stability theory of difference approximations for mixed initial boundary value problems II. Math. Comp. 26, 649-686.

Karniadakis, G. E. \& Triantafyllou, G. S. 1989 Frequency selection and asymptotic states in laminar wakes. J. Fluid Mech. 199, 441-469.

MACCoRMACK, R. W. 1969 The effect of viscosity in hypervelocity impact cratering. AIAA Paper 69-354.

OSHER, S. 1969 Systems of differential equations with general homogeneous boundary conditions. Trans. Amer. Math. Soc. 137, 177-201.

Rosнко, A. 1953 On the development of turbulent wakes from vortex streets. NACA TN-2913.

RUDY,D. H. \& STRIKWERDA, J. C. 1980 A nonreflecting outflow boundary condition for subsonic Navier-Stokes calculations. J. Comp. Phys. 36, 55-70.

Ruelle, D. \& Takens, F. 1971 On the nature of turbulence. Commun. Math. Phys. 20, 167-192.

\footnotetext{
${ }^{3}$ Private communication
} 
Sirovich, L. 1985 The Karman vortex trail and flow behind a circular cylinder. Phys. Fluids. 28, 2723-2726.

Sreenivasan, K. R. 1985 Transition and turbulence in fluid flows and low-dimensional chaos. In Frontiers in Fluid Mechanics (ed. S. H. Davis \& J. L. Lumley), 41-67. Springer.

SReEnivasan, K. R. 1989 Whither turbulence? Remarks on the 'dynamical systems approach' to the turbulence problem. In Turbulence at Cross Roads (ed. J. L. Lumley). Springer. To appear.

Townsend, J. C., Rudy, D. H. \& SiRovich, L. Computation and analysis of a cylinder wake flow. In Forum on Unsteady Flow Separation. (ed. K. N. Ghia), 165-174. ASME.

VANATTA, C. W. \& GHARIB, M. 1987 Ordered and chaotic vortex streets behind circular cylinders at low Reynolds numbers. J. Fluid Mech. 174, 113-133. 


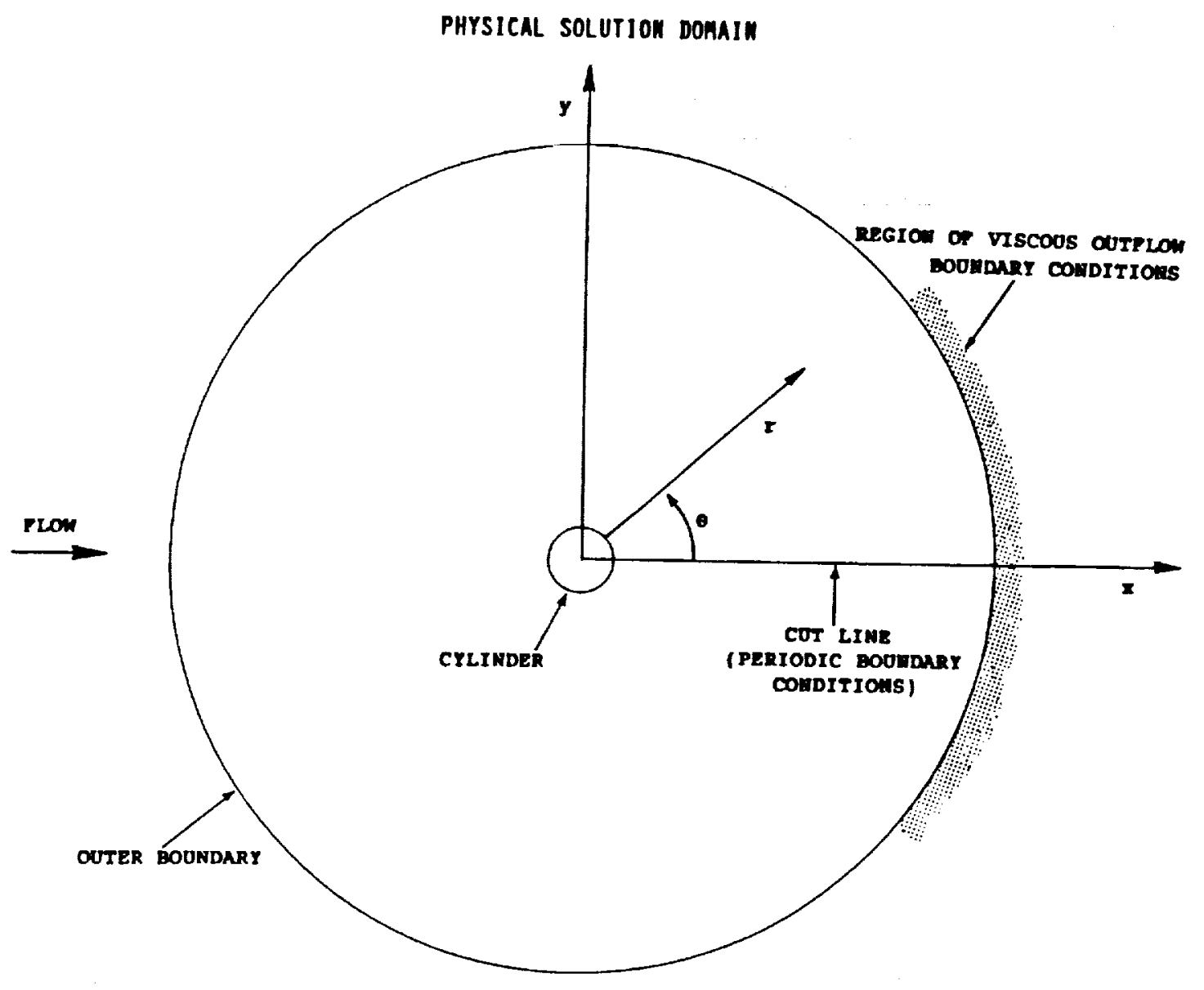

FIGURE 1. Solution domain in physical plane. 


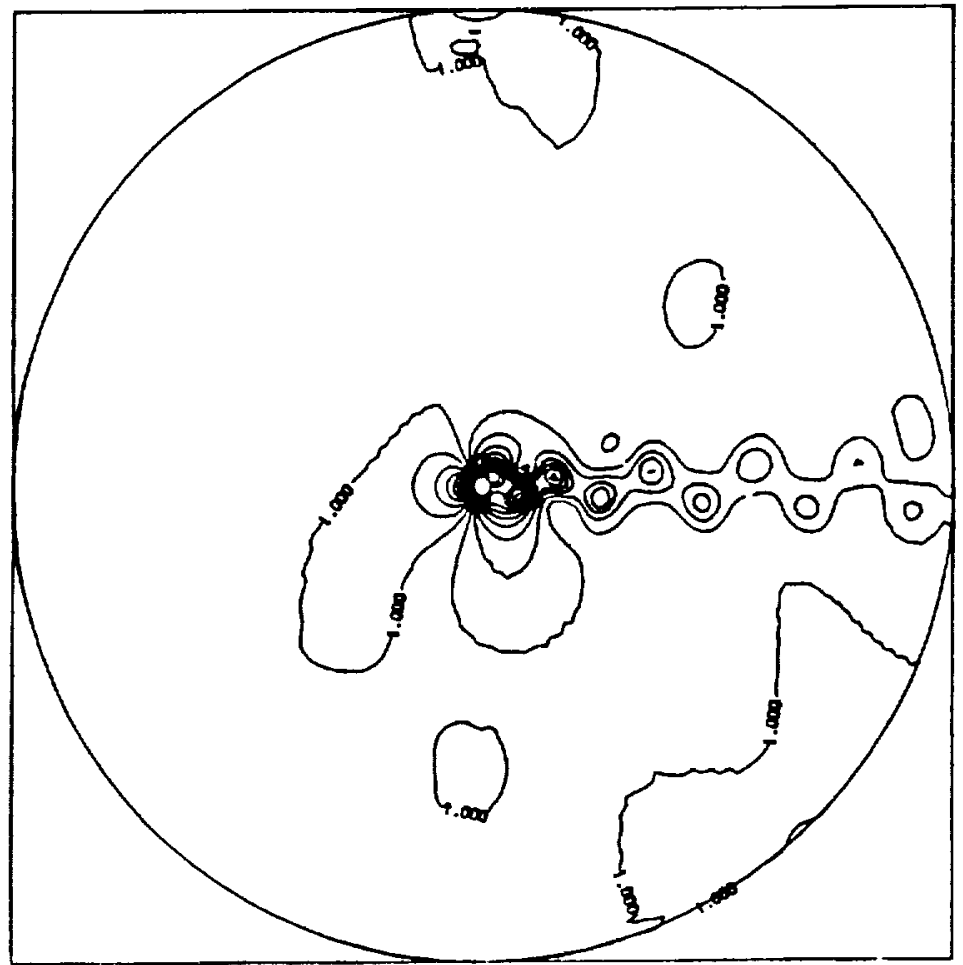

(a.) Primitive-variable boundary conditions.

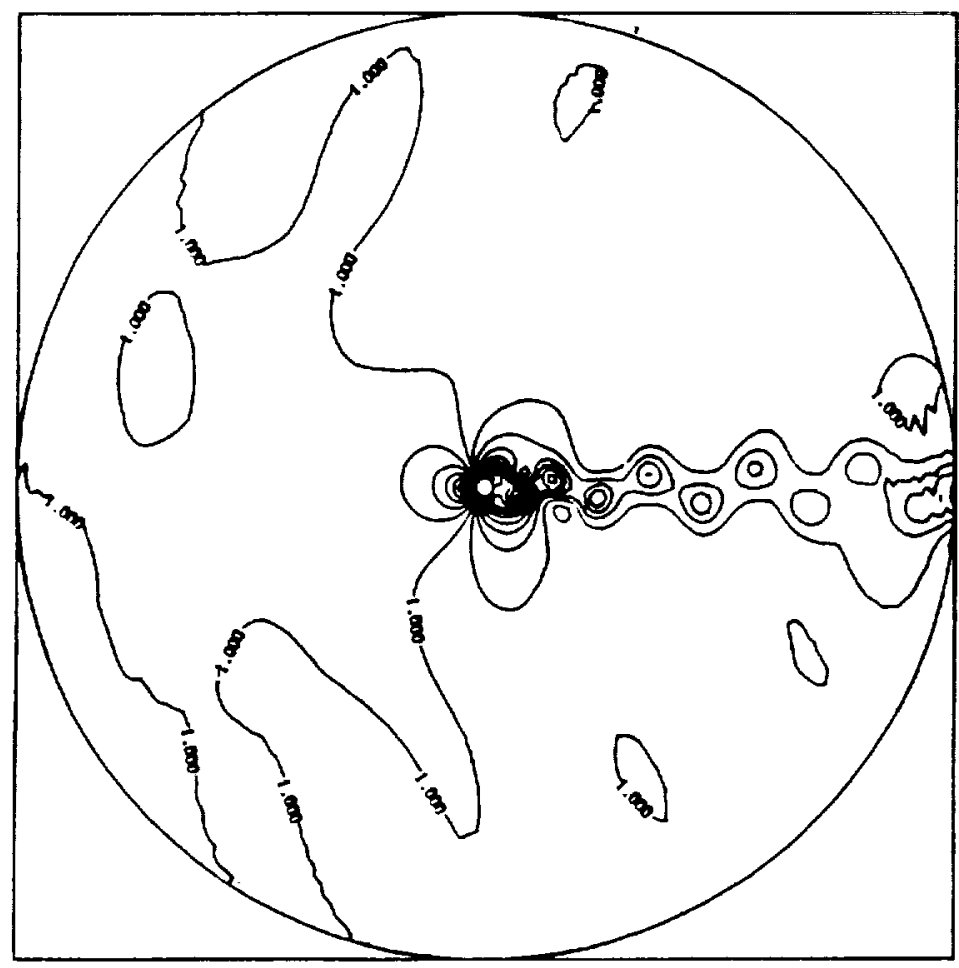

(b.) Characteristic-variable boundary conditions.

FIGURE 2. Lines of constant pressure $\left(\frac{p}{p_{\infty}}\right)$. Finite-difference code. Contour increment $=0.01 . M_{\infty}=0.4, R e_{\infty, D}=80$. 


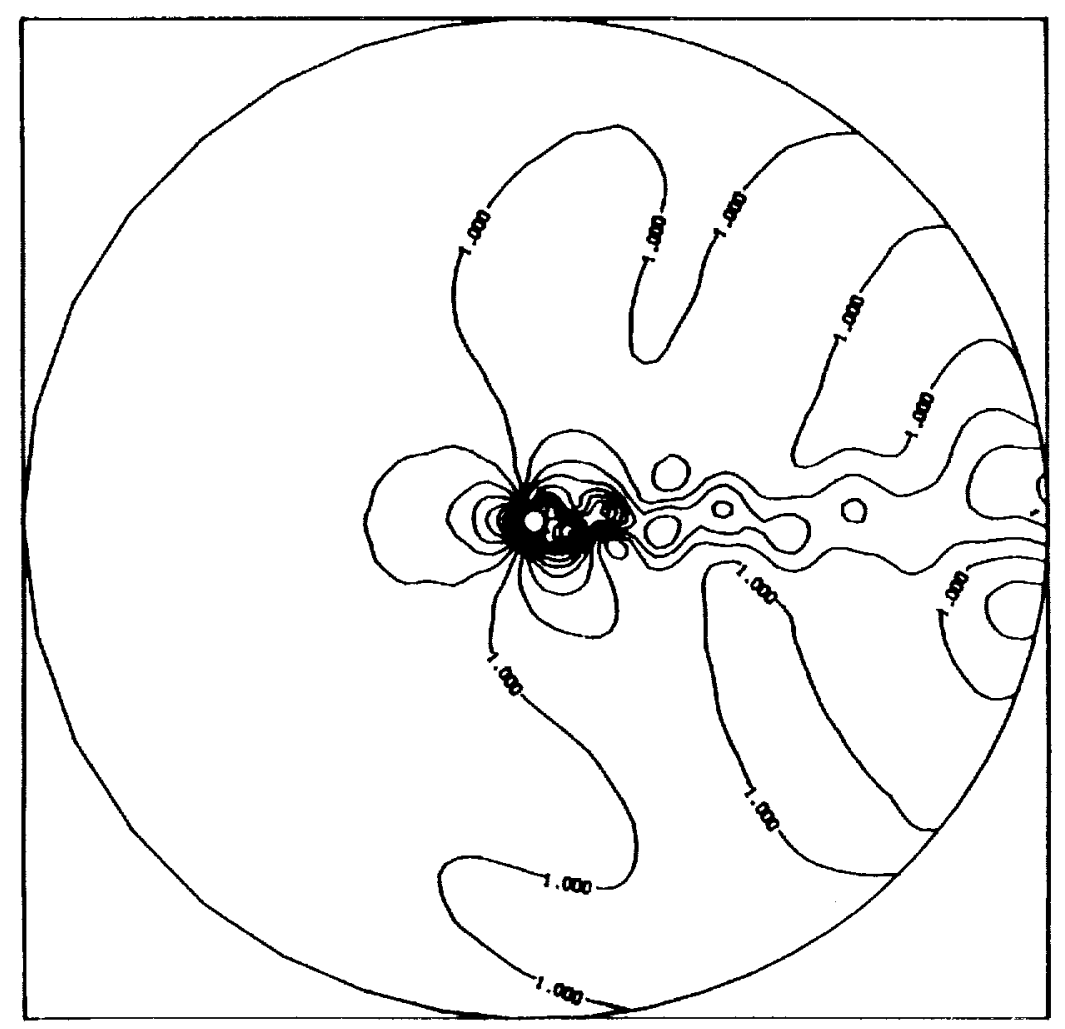

FIGURE 3. Lines of constant pressure $\left(\frac{p}{p_{\infty}}\right)$. Fully-spectral code. Contour increment $=0.01 . M_{\infty}=0.4, R e_{\infty, D}=80$. 


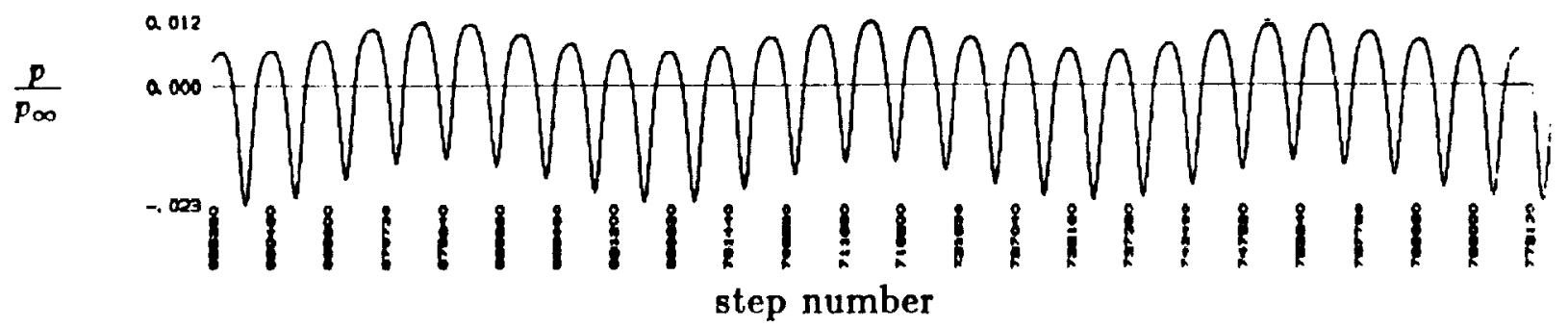

Primitive-variable boundary conditions.

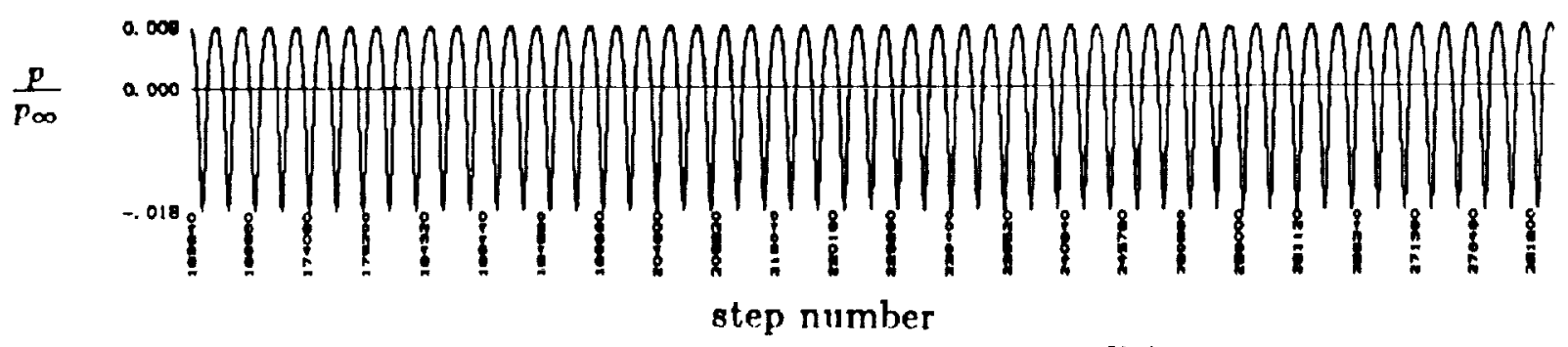

Characteristic-variable boundary conditions.

(a.) Time history of pressure.

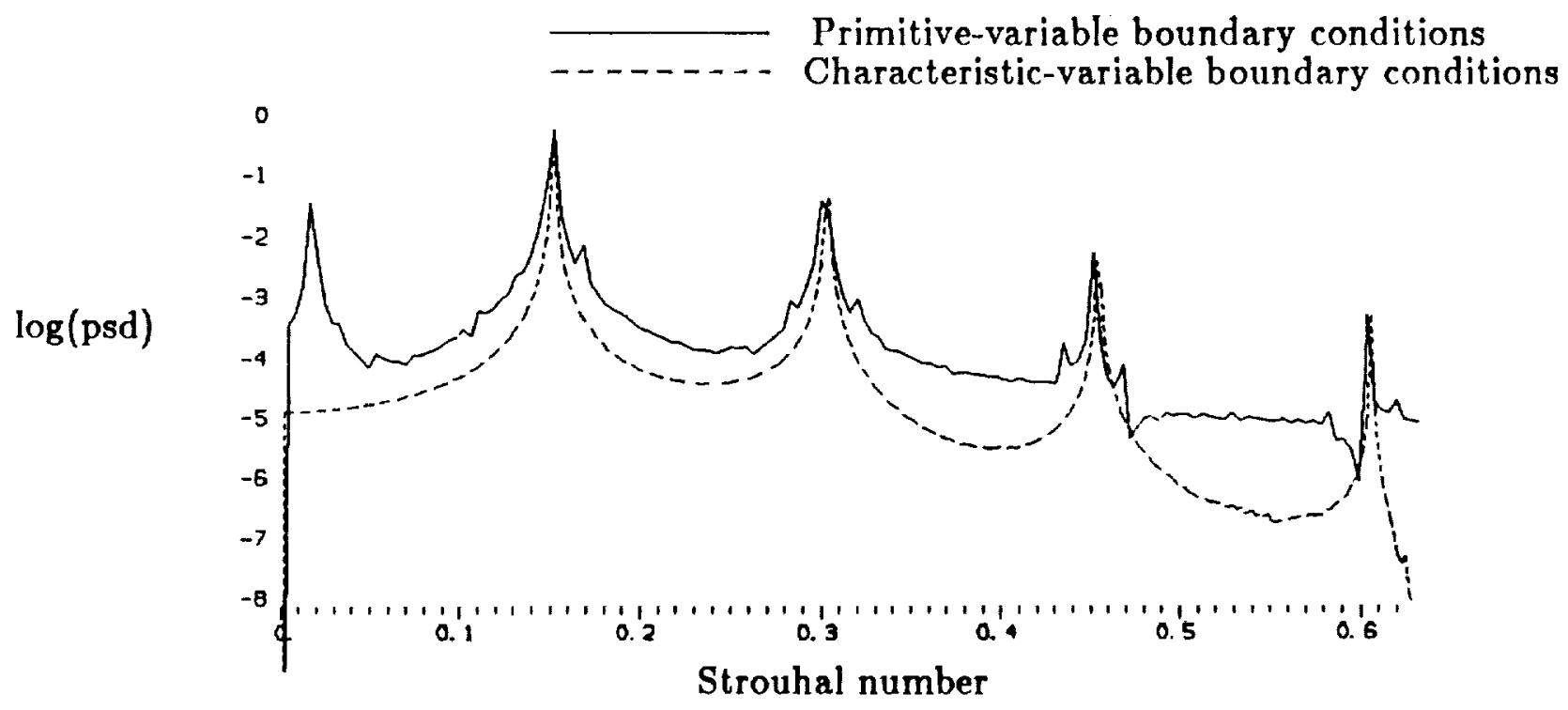

(b.) Frequency spectrum of pressure.

FIGURE 4. Effect of boundary conditions on pressure in wake region at location 10 cylinder diameters downstream of cylinder and one diameter above wake centerline. Finitedifference code. psd = power spectral density. 


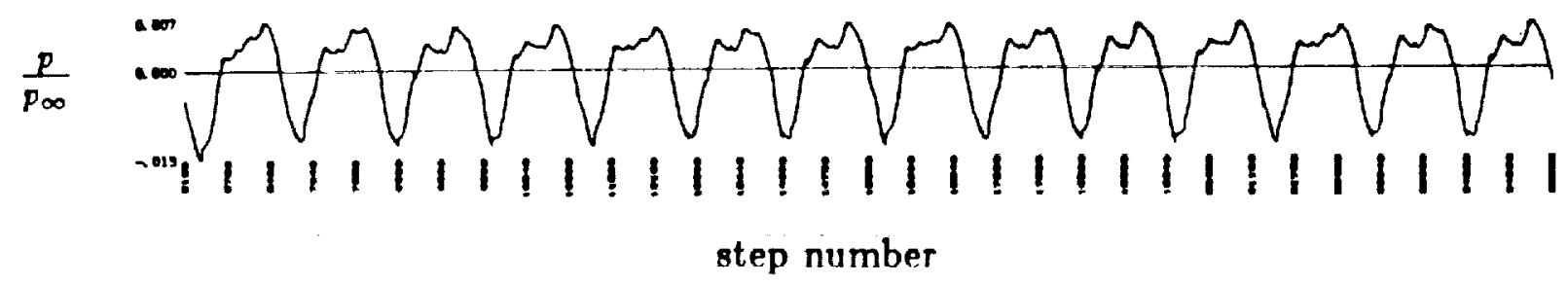

(a.) Time history of pressure.

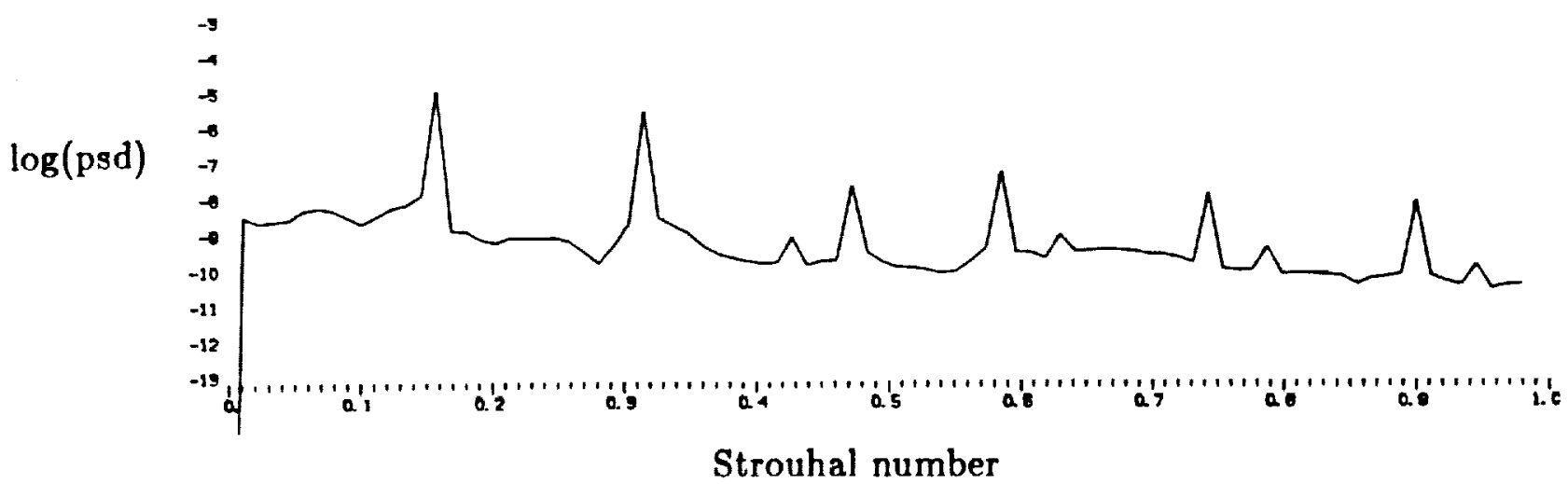

(b.) Frequency spectrum of pressure.

FIGURE 5. Computed pressure in wake region at location 10 cylinder diameters downstream of cylinder and one diameter above wake centerline. Fully-spectral code. psd = power spectral density. 


\begin{tabular}{|c|c|c|c|}
\hline \multicolumn{4}{|c|}{ Report Documentation Page } \\
\hline $\begin{array}{l}\text { 1. Report No. } \\
\text { NASA CR-181998 } \\
\text { ICASE Report No. } 90-16\end{array}$ & 2. Government Accession No. & \multicolumn{2}{|c|}{ 3. Recipient's Catalog No. } \\
\hline \multirow{2}{*}{\multicolumn{2}{|c|}{$\begin{array}{l}\text { 4. Title and Subritle } \\
\text { SECONDARY FREQUENCIES IN THE WAKE OF A CIRCULAR } \\
\text { CYLINDER WITH VORTEX SHEDDING }\end{array}$}} & \multicolumn{2}{|c|}{$\begin{array}{l}\text { 5. Report Date } \\
\text { February } 1990\end{array}$} \\
\hline & & \multicolumn{2}{|c|}{ 6. Performing Organization Code } \\
\hline \multirow{3}{*}{\multicolumn{2}{|c|}{$\begin{array}{l}\text { 7. Author(s) } \\
\text { Saul S. Abarbanel } \\
\text { Wai Sun Don } \\
\text { David Gottlieb } \\
\text { David H. Rudy } \\
\end{array}$}} & \multicolumn{2}{|c|}{$\begin{array}{l}\text { 8. Performing Organization Report No. } \\
90-16\end{array}$} \\
\hline & & \multirow{2}{*}{\multicolumn{2}{|c|}{$\begin{array}{l}\text { 10. Work Unit No. } \\
505-90-21-01\end{array}$}} \\
\hline & & & \\
\hline \multirow{2}{*}{\multicolumn{2}{|c|}{$\begin{array}{l}\text { Institute for Computer Applications in Science } \\
\text { and Engineering } \\
\text { Mail Stop 132C, NASA Langley Research Center } \\
\text { Hampton, VA 23665-5225 }\end{array}$}} & \multirow{3}{*}{\multicolumn{2}{|c|}{$\begin{array}{l}\text { 11. Contract or Grant No. } \\
\text { NAS } 1-18107 \\
\text { NAS } 1-18605 \\
\text { 13. Type of Report and Period Covered } \\
\text { Contractor Report }\end{array}$}} \\
\hline & & & \\
\hline \multirow{2}{*}{\multicolumn{2}{|c|}{$\begin{array}{l}\text { 12. Sponsoring Agency Name and Address } \\
\text { National Aeronautics and Space Administration } \\
\text { Langley Research Center } \\
\text { Hampton, VA 23665-5225 }\end{array}$}} & & \\
\hline & & \multicolumn{2}{|c|}{ 14. Sponsoring Agency Code } \\
\hline \multicolumn{4}{|l|}{ 15. Supplementary Notes } \\
\hline \multicolumn{2}{|l|}{$\begin{array}{l}\text { Langley Technical Monitor: } \\
\text { Richard W. Barnwel1 }\end{array}$} & \multicolumn{2}{|c|}{$\begin{array}{l}\text { ubmitted to Journal of } \\
\text { Fluid Mechanics }\end{array}$} \\
\hline \multicolumn{4}{|l|}{ 16. Abstract } \\
\hline \multicolumn{4}{|c|}{$\begin{array}{l}\text { A detailed numerical study of two-dimensional flow past a circular cylinder } \\
\text { at moderately low Reynolds numbers has been conducted using three different numer- } \\
\text { ical algorithms for solving the time-dependent compressible Navier-Stokes equa- } \\
\text { tions. It was found that if the algorithm and associated boundary conditions were } \\
\text { consistent and stable, then the major features of the unsteady wake were well- } \\
\text { predicted. However, it was also found that even stable and consistent boundary } \\
\text { conditions could introduce additional periodic phenomena reminiscent of the type } \\
\text { seen in previous wind-tunnel experiments. However, these additional frequencies } \\
\text { were eliminated by formulating the boundary conditions in terms of the character- } \\
\text { istic variables. An analysis based on a simplified model provides an explanation } \\
\text { for this behavior. }\end{array}$} \\
\hline \multirow{2}{*}{$\begin{array}{l}\text { 17. Key Words (Suggested by Author(s)) } \\
\text { Navier-Stokes, Low Reynolds numbers, } \\
\text { cylinder, CFD, shedding frequencies, } \\
\text { boundary conditions }\end{array}$} & \multicolumn{3}{|c|}{ 18. Distribution Statement } \\
\hline & $\begin{array}{l}02 \\
34 \\
\text { Unc }\end{array}$ & $\begin{array}{l}\text { Mechanics } \\
\text { ed - Unlimit }\end{array}$ & \\
\hline $\begin{array}{l}\text { 19. Security Classif. (of this report } \\
\text { Unclassified }\end{array}$ & is page) & $\begin{array}{l}\text { 21. No. of pages } \\
26\end{array}$ & $\begin{array}{r}\text { 22. Price } \\
\mathrm{AO} 3\end{array}$ \\
\hline
\end{tabular}


